\title{
A Simplified Approach: Teaching PERT with Ease and without Drawing Networks
}

\author{
Zhaobo Wang \\ Correspondence: Zhaobo Wang, 285 Madison Ave., Fairleigh Dickinson University, Madison NJ 07940, USA
}

Received: September 8, 2015

Accepted: September 16, $2015 \quad$ Online Published: November18, 2015

doi:10.11114/jets.v4i1.1073

URL: http://dx.doi.org/10.11114/jets.v4i1.1073

\begin{abstract}
Program Evaluation and Review Technique (PERT) has been widely used in all industries and taught in Project Management courses for more than 50 years. Hall, N.G. (2015, p. 945) points out: "The practice of Project Management has expanded exponentially in the last 15 years," but this rapid development has not been matched "by a corresponding increase either in research activities, or in the training of academic researchers in project management. The mismatch is creating significant opportunities for academic research management..." This paper provides two tips for teaching PERT for the Project Management or Operations-Management courses: 1) the proposed simplified formulas which can be used in classrooms effectively since they do not require division or truncation when calculating the expected duration and variance. The formulas not only simplifies the calculations, but also generates more accurate results (demonstrated through simulations) than the traditional approach of using truncated estimations; and 2) An approach to finding critical and non-critical activities without drawing a network chart so that a relatively large project can be efficiently demonstrated in a classroom setting. The paper demonstrates that by using only a table and avoiding the graphical presentation of a complicated network diagram, instructors can show relatively larger projects on blackboards and students, particularly those who prefer logic table calculations to drawing graphical networks, can better understand and master the PERT technique.
\end{abstract}

Keywords: PERT, teaching project management

\section{Introduction}

This paper offers an alternative approach to teaching a business process known as project management. The Project Management Institution (PMI) defines a project as "a temporary endeavor undertaken to create a unique product, service, or result" (PMI, 2013). The use of a project approach has long been applied in the construction industry. Now, it has spread to nearly all avenues of work. A modern-day example of this can be found nearly anywhere; for instance, when Pope Francis visited the United States in September of 2015, every aspect of his trip was carefully planned by a project management team. CBS News described this event as "unprecedented," calling it "the largest security operation in U.S. history" (CBS News, September 22, 2015). At its core, this is the nature of project management: planning for unique situations, taking into consideration every aspect of a project. A project management team must establish objectives, estimate a lifespan with a clearly defined beginning and ending, and identify all of the relevant subordinate activities, as well as determine the relative priority of and the appropriate sequencing among these activities. The activities of a project are interrelated and there are precedence relationships among the activities, which involve constraints that require one or more tasks to be completed before another activity can start. The objectives of a project management team are typically for the project to be completed on-time and on-budget, meeting or exceeding other specific performance requirements. Although a project management approach can be traced all the way back to the building of the Egyptian pyramids, and its predominant application type has its roots in engineering and construction projects, the major methodological achievements in the project management occurred in the 1960s when CPM (Critical Path Method) and PERT (Program Evaluation and Review Technique) were almost simultaneously developed.

Malcolm, Roseboom, Clark, and Fazar (1959) developed the Program Evaluation and Review Technique (PERT) for the Polaris Weapon System. In conjunction with the Critical Path Method (CPM), developed jointly by the Du Pont Company and Remington Rand (Walker and Sayer, 1959) and further expanded by Kelley (1961), PERT has been taught in classroom and used in practice for over 50 years. It is particularly useful when the durations of the various activities associated with a project are uncertain. PERT treats these durations for carrying out an activity as variables. 
The duration variables, due to the nature of uncertainty and within a range, are assumed to be the beta distribution which requires a cumbersome process for the determination of a solution to a cubic equation. So proponents of PERT suggested using an approximation formula and proved empirically that these formula yielded results that were close to the results yielded by the more complex algorithm and computations based on the beta distribution (Archibald and Villoria, 1967, p. 449.)

An alternate approach to approximating the beta distribution which is undertaken by PERT is to develop an approach to directly simulate the beta distribution of durations. Schonberger (1981), however, suggested that project managers may be better off by taking advantage of the insights gained from PERT's simpler approximation approach, which would avoid the need for simulating the distribution of durations and undertaking the complex calculations associated with solving the cubic equations. In addition, Kamburowski (1996) defended and validated the PERT's formula of calculating mean and variance. Davis (2008, p. 139) argued, "There has been a great deal of confusion and misunderstanding in the literature about how to carry out project simulations using the beta distribution based on the PERT paradigm". But Davis's own "PERT-Beta distribution" may also be too complex to be understood by students taking a project management course and even too complex to be practical for many practitioners. Roman (1962) tried to further simplify the use of PERT by developing an approximation procedure which decreased the number of relevant parameters to be estimated from three to two thereby focusing only the most likely and pessimistic times. However, Cottrell (1999) tested Roman's simplified PERT duration formula and found out that the results are "subject to errors of greater than $10 \%$ when the skewness of the actual distribution is greater than 0.28 or less than $-0.48 . "$

This evolution in the development and understanding of PERT has led us to the current situation, whereby all standard project management text books are still based on the PERT's original three parameters estimation approach (such as Larson and Cray, 2013, Project Management Institute (2013), Kerzner (2013), Klastorin (2010), and Milosevic (2003)). Therefore, the purpose of this paper is to allow instructors to further simplify the PERT formula in the classroom so that students - future project managers - can gain a better understanding of the usage of PERT for a relatively large project without even using a calculator or computer, just the blackboard! The paper also demonstrates that the simplified method not only improves the accuracy but also shows a nature of the robustness in PERT formula.

\section{Teaching Method 1}

\subsection{Traditional PERT Formula}

The project management team, after clearly defining the scope and deliverable objectives of a project, needs to successively subdivide the project into smaller and smaller work elements. This is called the Work Breakdown Structure (WBS). This breakdown process is repeated until the details of the component elements are small enough to be manageable. The smallest elements in the WBS are called activities. Every activity needs a duration and cost estimate. Unlike the CPM, which deals with activities whose duration and cost can be relatively easy to estimate, PERT deals with unique or non-routine or unprecedented projects, such as a groundbreaking research project, wherein accurate estimations of each activity's duration and cost are almost impossible. PERT makes these estimations possible by requiring experts to provide three estimated times for each of the activities: Let $\boldsymbol{a}$ represent the activity's duration with the most optimistic (least) time, i.e., assuming the best possible scenario (i.e., under the most favorable conditions, even if chance of such favorable conditions is less than one percent); $\boldsymbol{m}$ as the most likely duration, i.e., the mode of all normal estimations of duration gathered from all the people involved; and $\boldsymbol{b}$ as the most pessimistic duration, i.e., the estimate of duration under the most unfavorable conditions, even if there was only a $1 \%$ or less chance that such unlucky conditions would be observed. Basically, according to the PERT, each activity duration will range from $\boldsymbol{a}$, the most optimistic time, to $\boldsymbol{b}$, the most pessimistic time. The probability distribution within this range is manifested in a beta distribution, with $\boldsymbol{m}$ as the mode. The activity duration may be skewed more towards the high $(\boldsymbol{b})$ and low $(\boldsymbol{a})$ ends of the data range. See chart $1 \mathrm{a}, 1 \mathrm{~b}$ and $1 \mathrm{c}$ for the three typical beta distributions: 


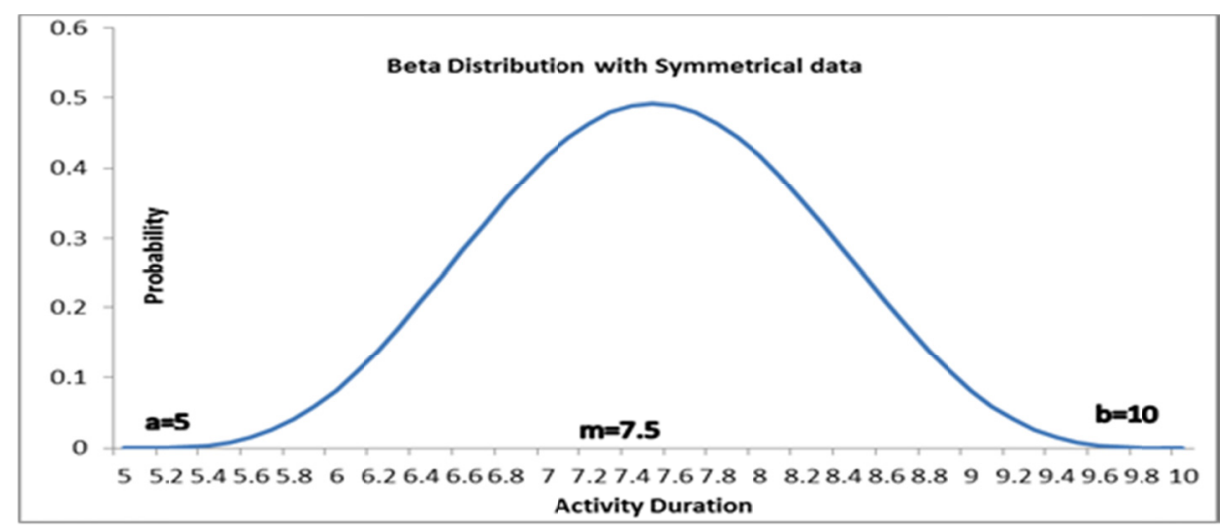

Chart 1a: Symmetrical Beta Distribution

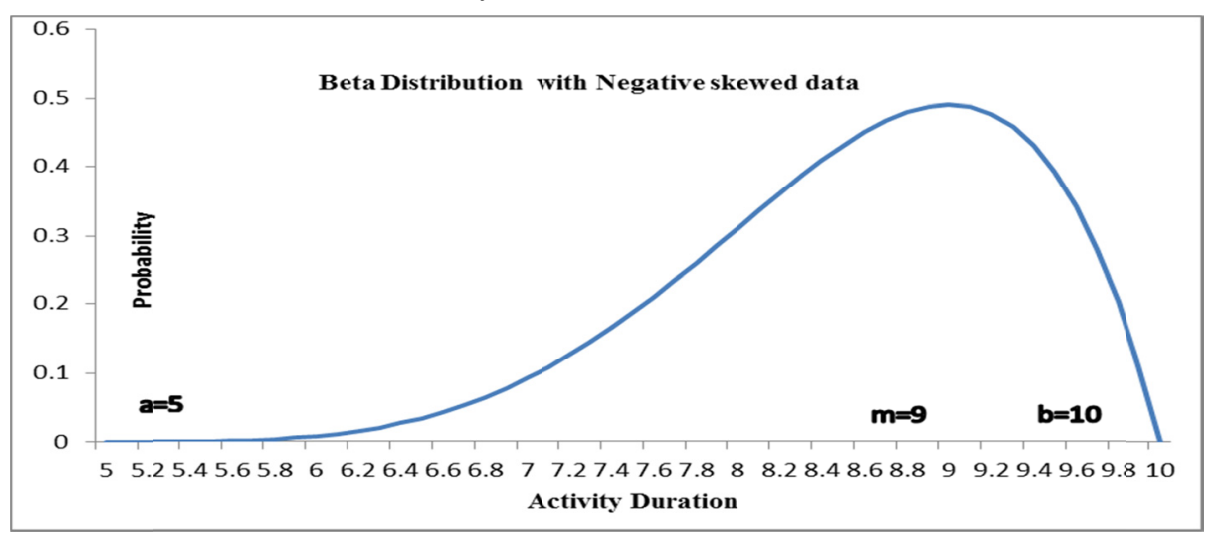

Chart 1b: Negative Skewed Beta Distribution

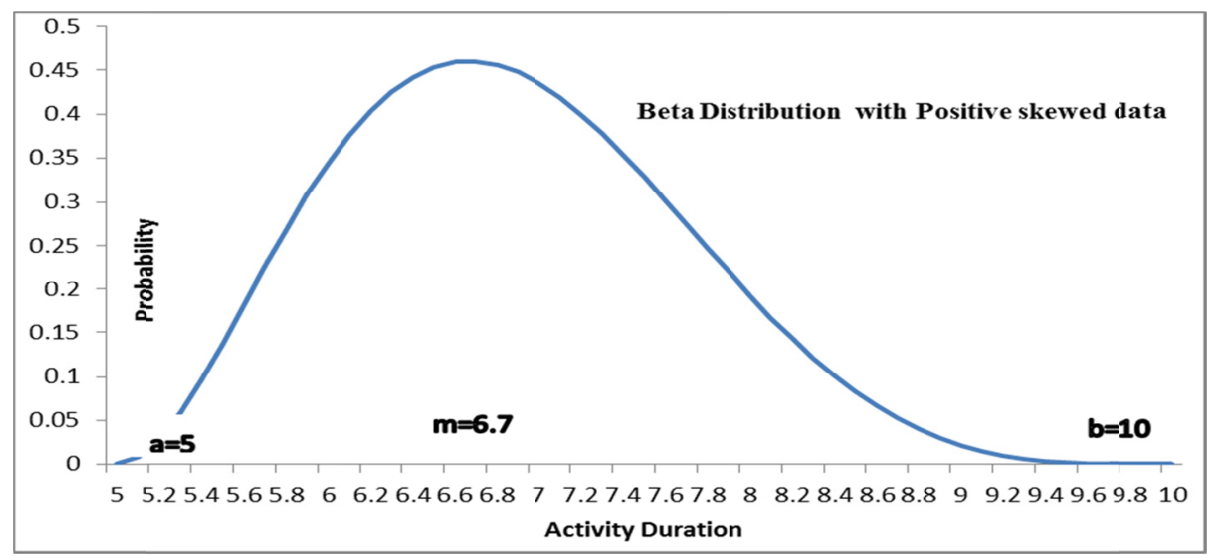

Chart 1c: Positive Skewed Beta Distribution

The skewed beta distribution creates a computational challenge. PERT, on the other hand, uses a simple arithmetic weighted average of the mode and the range as the expected duration for each activity, where the mode (m) carries $2 / 3$ of the weight and the two extreme duration times ( $\boldsymbol{a}$ and $\boldsymbol{b}$ ) share the remaining $1 / 3$ of the weight equally. Clark stated that by empirical numerical simulations, the results obtained from the complicated beta distribution and the results from the simple weighted average approach "are closely approximated" (1962, p. 406).

On the other hand, PERT assumes the standard deviation of an activity's duration is one-sixth of the range. The rationale is that the beta distribution can be approximated as a truncated normal distribution where more than $99 \%$ of the duration variations with all possible probabilistic outcomes are within three-standard-deviations from the mean, or six-standard-deviation within the range, i.e., the standard deviation of the duration is $(\boldsymbol{b}-\boldsymbol{a}) / 6$. Kamburowski (1996) defended the PERT simple estimates of the weighted average as the expected duration and the one-sixth of the range as the standard deviation by presenting a theoretical validation of them. His results show that the maximum absolute error for the mean is less than $3.87 \%$ of the range $(\boldsymbol{b}-\boldsymbol{a})$, and the maximum absolute error for the standard deviation is less than $2.58 \%$ of the range $(\boldsymbol{b}-\boldsymbol{a})$. 
PERT's approximated formulas of calculating the mean and standard deviation (variance), after half a century and withstanding many criticisms such as the criticisms offered by Keefer and Vardini (1993), are still standing tall: the PERT's mean and variance can be found in almost every textbook in the areas of Operations Research, Management Science, Operations Management and Supply Chain Management. PERT's formulas are employed in almost all project management software algorithms.

These two famous formulas of the expected duration $t_{i}$ and the variance $\operatorname{Var}_{i}$ are represented in equation (1) and (2) as follows, where $\mathrm{i}$ represents one of the $\mathrm{n}$ activities in a project:

$$
\begin{aligned}
& \mathrm{t}_{\mathrm{i}}=\left(4 \boldsymbol{m}_{\mathrm{i}}+\boldsymbol{a}_{\mathrm{i}}+\boldsymbol{b}_{\mathrm{i}}\right) / 6 \\
& \operatorname{Var}_{\mathrm{i}}=\left[\left(\boldsymbol{b}_{\mathrm{i}}-\boldsymbol{a}_{\mathrm{i}}\right) / 6\right]^{2}
\end{aligned}
$$

\subsection{Modified PERT Formula}

Each of these two equations involves a division by 6 or $6^{2}$. When demonstrating the calculations in class, decimals and truncations have to be used and that practice is not computationally convenient. Students may focus too much on the calculation involving the accuracy of the decimals to see the bigger picture. The teaching tip offered by this paper suggests a way to avoid the decimals and truncations: don't do the division; just keep the fraction with denominator of 6 for $t_{i}$ and $6^{2}$ for $\operatorname{Var}_{i}$. Thus the above two equations can be written as:

$$
\begin{aligned}
& \mathrm{T}_{\mathrm{i}}=6 \mathrm{t}_{\mathrm{i}}=\left(4 \boldsymbol{m}_{\boldsymbol{i}}+\boldsymbol{a}_{\boldsymbol{i}}+\boldsymbol{b}_{\mathrm{i}}\right) \\
& \mathrm{V}_{\mathrm{i}}=6^{2} \operatorname{Var}_{\mathrm{i}}=\left(\boldsymbol{b}_{\boldsymbol{i}}-\boldsymbol{a}_{\boldsymbol{i}}\right)^{2} .
\end{aligned}
$$

The benefits of not dividing the right hand side of both equations by 6 and $6^{2}$ are quite obvious:

- Students can easily calculate the new expected durations using the modified equation (3) with only multiplication and addition.

- The expected duration $\mathrm{T}_{\mathrm{i}}$ is then used to calculate the Earliest Start Time $\left(\mathrm{ES}_{\mathrm{i}}\right)$, Earliest Finish Time $\left(\mathrm{EF}_{\mathrm{i}}\right)$, Latest Finish Time $\left(\mathrm{LF}_{\mathrm{i}}\right)$, and Latest Start Time $\left(\mathrm{LS}_{\mathrm{i}}\right)$ in order to find out which activities are critical and which are not. For each of the non-critical activities, the slack time (the difference between the latest start time and the earliest start time) is also calculated in order to know the flexibility the project manager can have for the activity.

- Furthermore, for each of the critical activities, the variance is calculated using equation (4) in order to find out the project's standard deviation.

- Under the traditional formulas (1) and (2), all these calculations are based on the truncated decimals. While the modified formulas (3) and (4) will generate only integer durations and variance if the original estimations ( $\boldsymbol{m}$, $\boldsymbol{a}, \boldsymbol{b})$ are integers, all these calculations (ES, EF, LF, LS, Slack, and variance) become much easier and no truncation is needed. In the classroom setting, it can be much more efficient to do this way, particularly when the example used on the blackboard has a relative large number of activities.

- The result by using formula (3) and (4) can be more accurate than (1) and (2) since no truncation is used.

Due to the greater computational simplicity, it can be argued that the modified approach is likely to be accepted by a larger proportion of students. Table 1 describes the simplicity of the modified formula and its calculation as an example project (without even using a calculator).

Table 1. Example of Using Modified Formula

\begin{tabular}{lllllll}
\hline Activity & $\begin{array}{l}\text { Immediate } \\
\text { Predecessor }\end{array}$ & $\boldsymbol{a}$ & $\boldsymbol{m}$ & $\boldsymbol{b}$ & $\mathrm{T}_{\mathrm{i}}=4 \boldsymbol{m}+\boldsymbol{a}+\boldsymbol{b}$ & $* \mathrm{~V}_{\mathrm{i}}=(\boldsymbol{b}-\boldsymbol{a})^{2}$ \\
\hline A & - & 4 & 6 & 7 & $=4 \times 6+4+7=35$ & $=(7-4)^{2}=3^{2}=9$ \\
B & - & 1 & 2 & 3 & $=4 \times 2+1+3=12$ & $=(3-1)^{2}=2^{2}=4$ \\
C & A & 6 & 6 & 6 & $=4 \times 6+6+6=36$ & $=(6-6)^{2}=0^{2}=0$ \\
D & A & 5 & 8 & 11 & $=4 \times 8+5+11=48$ & $=(11-5)^{2}=6^{2}=36$ \\
E & B,C & 1 & 9 & 18 & $=4 \times 9+1+18=55$ & $=(18-1)^{2}=17^{2}=289$ \\
F & D & 2 & 3 & 6 & $=4 \times 3+2+6=20$ & $=(6-2)^{2}=4^{2}=16$ \\
G & D & 1 & 7 & 8 & $=4 \times 7+1+8=37$ & $=(8-1)^{2}=7^{2}=49$ \\
H & E,F & 4 & 4 & 6 & $=4 \times 4+4+6=26$ & $=(6-4)^{2}=2^{2}=4$ \\
I & G,H & 1 & 6 & 8 & $=4 \times 6+1+8=33$ & $=(8-1)^{2}=7^{2}=49$ \\
J & I & 2 & 5 & 7 & $=4 \times 5+2+7=29$ & $=(7-2)^{2}=5^{2}=25$ \\
K & I & 8 & 9 & 11 & $=4 \times 9+8+11=55$ & $=(11-8)^{2}=3^{2}=9$ \\
L & J & 2 & 4 & 6 & $=4 \times 4+2+6=24$ & $=(6-2)^{2}=4^{2}=16$ \\
M & J & 1 & 2 & 3 & $=4 \times 2+1+3=12$ & $=(3-1)^{2}=2^{2}=4$ \\
N & L,M & 6 & 8 & 10 & $=4 \times 8+6+10=48$ & $=(10-6)^{2}=4^{2}=16$ \\
\hline
\end{tabular}

$* \mathrm{~V}_{\mathrm{i}}$ can be delayed to calculate until the critical activities are identified 


\section{Teaching Method 2}

\subsection{Finding the Critical Activities without Drawing the CPM Network}

For the example project in table 1 with 14 activities, drawing the CPM network to calculate the ES, EF, LS, and LF as well as the slack time for each activity on the blackboard is time consuming and may not meaningfully add to the understanding of PERT for some students who prefer logical thinking to visual presentation of a network diagram. This paper suggests simply using the table (not drawing the network) to calculate all the times needed to find the critical activities and path:

The procedure that students need to find the ES (Earliest Start time) is:

1) For those activities without immediate predecessors, their $E S=0$ (activity $A$ and $B$ in the example);

2) For those activities with only one immediate predecessor, ES = EF of its immediate predecessor, such as activity $\mathrm{C}, \mathrm{D}, \mathrm{F}, \mathrm{G}, \mathrm{J}, \mathrm{K}$, and $\mathrm{M}$;

3) For those activities with more than one immediate predecessors, ES = largest EF among all of their immediate predecessors), such as $\mathrm{E}, \mathrm{H}, \mathrm{I}$ and $\mathrm{N}$;

4) All EFs are simply the addition of its ES and expected duration: $E F_{i}=E S_{i}+T_{i}$.

After calculating the ES and EF for all activities, the procedures that students need to find the LF (Latest Finishing time) and LS (Latest Start time) are:

5) For those activities which are not in the column of immediate predecessors, i.e., the last activities in the project, their $\mathrm{LF}=$ the largest EF in the EF column. In the example case, activities $\mathrm{N}$ and $\mathrm{K}$ (students need to count A, B, C, and all the way to $\mathrm{N}$ from the immediate predecessors column to realize that $\mathrm{N}$ and $\mathrm{K}$ are not in the column);

6) For those activities that appear in the immediate predecessor column only once, LF $=$ LS of the successor; this applies to all activities B, C, E, F, G, H, L, and M.

7) For those activities that appear in the immediate predecessor column more than once, LF = smallest LS among those successors. This applies to activities J, I, D, and A;

8) All LSs are simply the subtraction of its LF and the expected duration: $\mathrm{LS}_{\mathrm{i}}=\mathrm{LF}_{\mathrm{i}}-\mathrm{T}_{\mathrm{i}}$.

9) Slack Time $=$ LS - ES or $=$ LF - EF.

Table 2. Illustrations of the Calculations without Drawing the Network

\begin{tabular}{|c|c|c|c|c|c|c|c|c|}
\hline Activity & $\begin{array}{l}\text { Immediate } \\
\text { Predecessor }\end{array}$ & $\mathrm{T}=4 \mathrm{~m}+\mathrm{a}+\mathrm{b}$ & ES & $\mathrm{EF}$ & LS & $\mathrm{LF}$ & Slack & $\mathrm{V}=(\mathrm{b}-\mathrm{a})^{\wedge} 2$ \\
\hline $\mathrm{A}$ & - & 35 & 0 & 35 & 0 & 35 & 0 & 9 \\
\hline B & - & 12 & 0 & 12 & 59 & 71 & $59 / 6$ & \\
\hline $\mathrm{C}$ & A & 36 & 35 & 71 & 35 & 71 & 0 & 0 \\
\hline D & A & 48 & 35 & 83 & 58 & 106 & $23 / 6$ & \\
\hline E & $\mathrm{B}, \mathrm{C}$ & 55 & 71 & 126 & 71 & 126 & 0 & 289 \\
\hline $\mathrm{F}$ & $\mathrm{D}$ & 20 & 83 & 103 & 106 & 126 & $23 / 6$ & \\
\hline G & $\mathrm{D}$ & 37 & 83 & 120 & 115 & 152 & $32 / 6$ & \\
\hline $\mathrm{H}$ & $\mathrm{E}, \mathrm{F}$ & 26 & 126 & 152 & 126 & 152 & 0 & 4 \\
\hline I & $\mathrm{G}, \mathrm{H}$ & 33 & 152 & 185 & 152 & 185 & 0 & 49 \\
\hline $\mathrm{J}$ & I & 29 & 185 & 214 & 185 & 214 & 0 & 25 \\
\hline K & I & 55 & 185 & 240 & 231 & 286 & $46 / 6$ & \\
\hline $\mathrm{L}$ & $\mathrm{J}$ & 24 & 214 & 238 & 214 & 238 & 0 & 16 \\
\hline $\mathrm{M}$ & $\mathrm{J}$ & 12 & 214 & 226 & 226 & 238 & $12 / 6$ & \\
\hline $\mathrm{N}$ & $\mathrm{L}, \mathrm{M}$ & 48 & 238 & 286 & 238 & 286 & 0 & 16 \\
\hline Total $==>$ & & & & & & 286 & & 408 \\
\hline
\end{tabular}

Only at the very end, the expected completion time is divided by 6 , and the critical path total variance is divided by 36 and then the square root of that quotient is taken to compute the critical path standard deviation. The slack times also need to be divided by 6 to reflect the correct time units.

\subsection{Finding the Total and Shared Slack Time from the Table Directly}

The slack time for each non-critical activity is the amount of time that the activity can slip without causing a delay in the project completion. The slack is based on the duration of the critical path. There are two kinds of slacks: Total slack and shared slack. When a non-critical activity's predecessor and successor are both critical, this activity's slack is the total slack. When a non-critical activity is the immediate predecessor of another non-critical activity, the slack time has to be shared between these two activities. That means, if the predecessor activity slips $n$ days, the successor activity's 
slack time will be reduced by the same $\mathrm{n}$ days. It seems that the project network method has an advantage in identifying the shared slack and total slack by the network graph. But we can also find them easily from the table method. Table 3 explains the way to trace and calculate the two kinds of slack times.

Activities B, D, F, G, K and M are non-critical activities since their slack times are not zero. B has no predecessor and its successor is E, which is a critical activity. Therefore, B's slack time is the total slack since B slips within its slack time will have no effect on the total project completion time. D's predecessor is a critical activity A. However, D has two non-critical successors, F and G. F and G's successors are critical activity $\mathrm{H}$ and I respectively. Thus, D has no total slack and its entire slack is shared slack, since any delay in D will reduce both F and G's slack times. Both F and G's total slack are the difference from D's slack. F and D have the same slack so F's total slack is also zero. But G's total slack is $32 / 6-23 / 6=9 / 6$. Activity K's predecessor is I, which is a critical activity. $\mathrm{K}$ has no successor. Thus, $\mathrm{K}$ enjoys its slack as the total slack. By the same token, we can easily trace the predecessor and successor of the activity M: its predecessor is J, which is a critical activity, and its successor is $\mathrm{N}$, which is also a critical activity. Therefore, M's slack is also the total slack.

Table 3. Illustrations of the Calculations of Shared and Total Slack Times Using Table Method

\begin{tabular}{lllll}
\hline Activity & $\begin{array}{l}\text { Immediate } \\
\text { Predecessor }\end{array}$ & Slack & $\begin{array}{l}\text { Total } \\
\text { Slack }\end{array}$ & $\begin{array}{l}\text { Shared } \\
\text { Slack }\end{array}$ \\
\hline A & - & 0 & & \\
B & - & $59 / 6$ & $59 / 6$ & 0 \\
C & A & 0 & & \\
D & A & $23 / 6$ & 0 & $23 / 6$ \\
E & B,C & 0 & & \\
F & D & $23 / 6$ & 0 & $23 / 6$ \\
G & D & $32 / 6$ & $9 / 6$ & $23 / 6$ \\
H & E,F & 0 & & \\
I & G,H & 0 & & \\
J & I & 0 & & \\
K & I & $46 / 6$ & $46 / 6$ & 0 \\
L & J & 0 & & \\
M & J & $12 / 6$ & $12 / 6$ & 0 \\
N & L,M & 0 & & \\
\hline
\end{tabular}

In summary, the table method can extend its usefulness to distinguish total slack and shared slack by simply tracing each non-critical activity's immediate predecessor(s) and successor(s). It can be easily proved that the slack time of a non-critical predecessor is not larger than the slack time of a non-critical successor. If its immediate predecessor and its immediate successor are both critical activities, the activity's slack time will be the total slack since it does not have to share the slack with any other activities. If its successor is also a non-critical activity, the first non-critical activity's slack will be all shared slack and the second non-activity's total slack will be the result of its slack minus the predecessor's shared slack.

\section{Results}

\subsection{Accuracy Comparisons}

To compare the rounding impact towards the completion measurements of the project for the example project from Table 2, the modified PERT method (No Rounding) is first used to calculate the baseline: the expected completion time for the project and the critical path standard deviation. The completion time with $90 \%$ probability is also calculated. Then, the traditional PERT calculations (equations (1) and (2)) are used three times, rounding to 0 , 1 , or 2 decimals respectively. The Excel function $\operatorname{ROUND}\left((\boldsymbol{a}+4 \boldsymbol{m}+\boldsymbol{b}) / 6\right.$, i) and $\operatorname{ROUND}\left(((\boldsymbol{b}-\boldsymbol{a}) / 6)^{\wedge} 2\right.$, i) are used to get the results, where $\mathrm{i}=0,1$, and 2 .

For the example project, if equations (1) and (2) are used and rounded for both the expected duration and the variance to 0 decimal place (i.e., integer), only the standard deviation has a relative large percent difference from the result that would apply if no rounding occurs (equation (3) and (4)). But the results are not that much different when they are rounded to 1 or 2 decimals. In any case, a more accurate result emerges based on this modified, simpler approach to complete the PERT process. 
Table 4. Comparison of Rounding Errors with the Example Project

\begin{tabular}{lllllll}
\hline & $\begin{array}{l}\text { Expected } \\
\text { Completion } \\
\text { Time }\end{array}$ & \% difference & $\begin{array}{l}\text { Critical Path } \\
\text { Standard } \\
\text { Deviation }\end{array}$ & $\begin{array}{l}\text { \% } \\
\text { difference }\end{array}$ & $\begin{array}{l}90 \% \\
\text { Complete } \\
\text { day }\end{array}$ & $\begin{array}{l}\% \\
\text { difference }\end{array}$ \\
\hline No Rounding & 47.67 & & 3.3665 & & 51.98 & \\
Round to 0 decimal & 48.00 & $0.69 \%$ & 3.1623 & $-6.07 \%$ & 52.05 & $0.13 \%$ \\
Round to 1 decimal & 47.60 & $-0.15 \%$ & 3.3615 & $-0.15 \%$ & 51.90 & $-0.15 \%$ \\
Round to 2 decimal & 47.66 & $-0.02 \%$ & 3.3645 & $-0.06 \%$ & 51.97 & $-0.02 \%$ \\
\hline
\end{tabular}

\subsection{Simulation Comparisons}

Of course, the accuracy percentage depends on the magnitude of the expected completion time which, in turn, depends on the magnitude of the three estimated times: $\boldsymbol{a}, \boldsymbol{m}$, and $\boldsymbol{b}$. A simulation is used by varying all $\boldsymbol{a}, \boldsymbol{m}$, and $\boldsymbol{b}: \boldsymbol{a}=$ $\operatorname{INT}(\operatorname{RAND}() * 10+1), \boldsymbol{m}=\boldsymbol{a}+\operatorname{INT}(\operatorname{RAND}() * 10)$ and $\boldsymbol{b}=\boldsymbol{m}+\operatorname{INT}(\operatorname{RAND}() * 10)$.

\subsubsection{Mean Completion Times}

With 100 simulation cases, the mean completion times with different rounding methods are shown in the Chart 2 . Only the "Round to 0 , blue color" can be seen in some cases. "Round to 1, red color" and "Round to 2, green color" are entirely covered by the "No Round, violet". This result indicates that round-error is not a problem for calculate the project expected completion time.

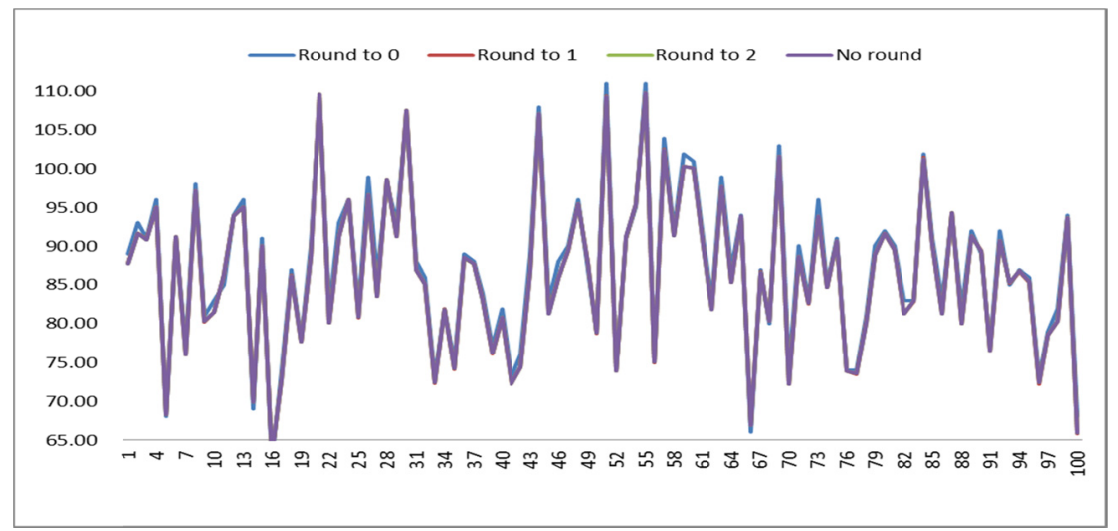

Chart 2. Mean Completion Times

The results of the percentage of the mean completion time difference are presented in Table 5:

Table 5. Percentage Difference of Mean Completion Time among Different Rounding

\begin{tabular}{lrrrr}
\hline Percentage Difference & $>1.50 \%$ & $>1.0 \%$ & $>0.5 \%$ & Largest \\
\hline Round to 0 decimals & $5 \%$ & $11 \%$ & $30 \%$ & $3.29 \%$ \\
Round to 1 decimals & $0 \%$ & $0 \%$ & $0 \%$ & $0.20 \%$ \\
Round to 2 decimals & $0 \%$ & $0 \%$ & $0 \%$ & $0.02 \%$ \\
\hline
\end{tabular}

Table 5 indicates that if the duration is rounded to 0 decimals, there are $5 \%$ of the simulation cases whose mean completion time is more than $1.5 \%$ higher or lower than the no-rounding mean completion time. The largest percentage difference is $3.29 \%$. 30\% of the simulation cases are at least $0.5 \%$ away from the correct and accurate results. Other than rounding to 0 decimal places, the simulation results seem to point to the robustness of the PERT for the mean completion time. If the calculation of expected duration and the critical activities' variances are just rounded to one decimal place, the resulting calculations are not very much different from what emerges from a calculation procedure where no rounding is done.

\subsubsection{Critical Path Standard Deviations}

However, the standard deviations may not be that insensitive with rounding decimal differences. Chart 3 shows clearly those differences are evident and Table 6 indicates the percentage differences: 


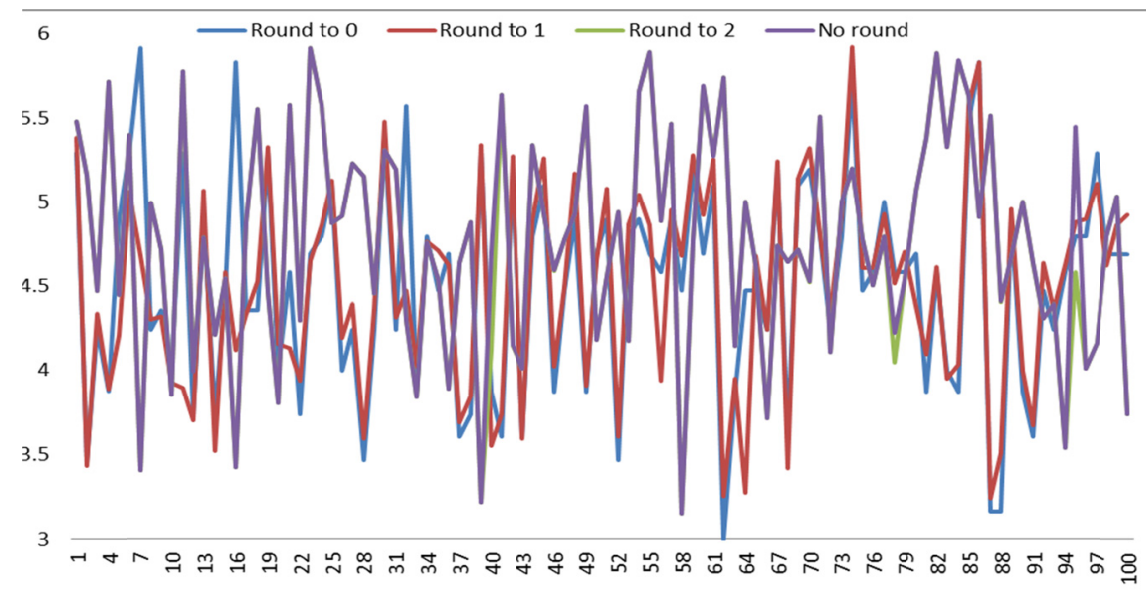

Chart 3 Standard Deviation's Variation

Table 6. Standard Deviation of Critical Path Difference

\begin{tabular}{llllll}
\hline Percentage Difference & $>40 \%$ & $>30 \%$ & $>20 \%$ & $>10 \%$ & Largest \\
\hline Round to 0 decimals & $5 \%$ & $11 \%$ & $30 \%$ & $59 \%$ & $73.62 \%$ \\
Round to 1 decimals & $4 \%$ & $14 \%$ & $31 \%$ & $61 \%$ & $65.85 \%$ \\
Round to 2 decimals & $0 \%$ & $0 \%$ & $1 \%$ & $3 \%$ & $15.96 \%$ \\
\hline
\end{tabular}

The differences are quite significant when each of the variances (equation (2)) is rounded to zero or one decimal. Table 6 shows that if the variance is rounded to 1 decimal places (second row), $61 \%$ of the simulation cases show at least $10 \%$ away from the correct and accurate results and the largest differential would be $65.85 \%$ ! Even the rounding to 2 decimals has 3 cases which lead to a differential of more than $10 \%$ (the largest one is $15.96 \%$ ). These results suggest that the variance calculation would need to be carried out to at least 2 to 3 decimal places in order to significantly reduce the differential.

\subsubsection{The Completion Times with 90\% Probability}

Lastly, suppose management would like to know the completion time with $90 \%$ confidence. It is calculated by the mean $+1.28 \times$ Standard Deviation. Table 7 shows the percentage difference comparing with the no-round (accurate) completion time with $90 \%$ confidence:

Table 7. Completion Time Difference with 90\% Probability

\begin{tabular}{lllll}
\hline & $>3 \%$ & $>2 \%$ & $>1 \%$ & Largest \\
Percentage Difference & & & & \\
\hline Round to 0 decimals & $4 \%$ & $14 \%$ & $48 \%$ & $5.25 \%$ \\
Round to 1 decimals & $3 \%$ & $13 \%$ & $43 \%$ & $3.31 \%$ \\
Round to 2 decimals & $0 \%$ & $0 \%$ & $1 \%$ & $1.22 \%$ \\
\hline
\end{tabular}

For rounding to 0 decimals, $48 \%$ of the cases are at least $1 \%$ shorter or longer than no-rounding result. For rounding to 1 decimal place, $43 \%$ of the cases are at least $1 \%$ shorter or longer than no-rounding result; the table shows only one case that is $1.22 \%$ shorter or longer than no-rounding results when rounding to 2 decimal places.

In conclusion, if the equations (1) and (2) are used, rounding to at least 2 decimal places is necessary to get the result close to the no-rounding result. If the proposed equations (3) and (4) are utilized, not only would the whole calculation become much easier, but a more accurate result is generated!

\section{Discussion}

The proposed modification of the PERT estimation process based on not dividing the mean duration by $6^{1}$ and not dividing the variance of the duration by $6^{2}$ and avoiding the often elaborate and confusing network diagram approach not only makes the teaching PERT easier, but also generates more accurate results. In addition, the table method used here also makes the demonstration of a relatively larger PERT project on the blackboard possible. The table approach can also identify the shared slack times among those non-critical activities without the drawing of network.

Another possible related research topic is to identify the second or third longest paths that may have larger standard deviations of the completion time. In other words, near-critical paths need to be studied closely as well. The simulation results in Table 5 seem to point to the robustness of the standard deviation such that the end result may not be very sensitive to a small change of the standard deviation, but further studies are needed.

Simplification of the beta distribution of the duration deserves a further study, since assumptions of the normal distribution may not reflect reality. In particular, teaching the beta distribution in a classroom setting and using the 
simulation creates another challenge, as well as an opportunity.

\section{References}

Archibald and Villoria (1967). Network-Based Management Systems (PERT/CPM). New York: John Wiley \& Sons. ISBN-13: 978-0471032502.

CBSNews (2015). Pope's visit triggers unprecedented security operation in U.S. http://www.cbsnews.com/news/pope-francis-us-visit-largest-security-operation-us-history/

Clark, C. E. (1962). The PERT model for the distribution of an activity time. Operations Research, 10, 405-406. http://dx.doi.org/10.1287/opre.10.3.405

Cottrell, W. (1999). Simplified Program Evaluation and Review Technique (PERT), Journal of Construction Engineering Management, 125(1), 16-22. http://dx.doi.org/10.1061/(ASCE)0733-9364(1999)125:1(16)

Hall, N. G. (2015). Handbook on Project Management and Scheduling, 2, 945-970.

Kamburowski, J. (1996). New Validations of PERT Times. Omega, International Journal of Management Science, 25(3), 323-328. http://dx.doi.org/10.1016/S0305-0483(97)00002-9

Keefer, D. L., \& Verdini, W. A. (1993). Better estimation of PERT activity time parameters. Management Science, 39, 1086-1091. http://dx.doi.org/10.1287/mnsc.39.9.1086

Kelley, J. (1961). Critical path planning and scheduling: mathematical basis. Operations Research, 9, 296-320. http://dx.doi.org/10.1287/opre.9.3.296

Kerzner, H. (2013). Project Management: A Systems Approach to Planning, Scheduling, and Controlling (8th ed.). Wiley. ISBN 0-471-22577-0.

Klastorin, T. (2010). Project Management: Tools and Trade-offs (3rd Ed.). Wiley, ISBN 978-0-471-41384-4

Larson, E. W., \& Clifford, F. C. (2013). Project Management: The Managerial Process, 6th Ed., McGraw-Hill/Irwin, 2013. ISBN-13: 978-1259186400.

Malcolm, D. G., Roseboom, J. H., Clark, C. E., \& Fazar, W. (1959). Application of a Technique for Research and Development Program Evaluation. Operations Research, 7(5), 646-669. http://dx.doi.org/10.1287/opre.7.5.646

Milosevic, D. Z. (2003). Project Management ToolBox: Tools and Techniques for the Practicing. Wiley. ISBN 978-0-471-20822-8.

Project Management Institute (2013). A Guide to the Project Management Body of Knowledge (5th Ed.). ISBN 978-1-935589-67-9.

Roman, D. D. (1962). The PERT System: An Appraisal of Program Evaluation Review Technique. ACAD MANAGE J., April 1 5(1), 57-65; http://dx.doi.org/10.2307/254602

Ron, D. (2008). Teaching Note-Teaching Project Simulation in Excel Using PERT-Beta Distributions. INFORMS Transactions on Education, 8(3), 139-148. http://dx.doi.org/10.1287/ited.1080.0013

Schonberger, R. J. (1981). Why projects are "always" late: A rationale based on manual simulation of a PERT/CPM network. Interfaces, 11(5), 66-70. http://dx.doi.org/10.1287/inte.11.5.66

Walker, M. R., \& Sayer, J. S. (1959), Critical Path Planning and Scheduling, Report 6959, Wilmington, DE: E.I. du Pont de Nemours \& Co., Inc, March 1959. http://dx.doi.org/10.1145/1460299.1460318

This work is licensed under a Creative Commons Attribution 3.0 License. 